\title{
Identification of an expanded CAG repeat in the Huntington's disease gene (IT15) in a family reported to have benign hereditary chorea
}

\author{
J C MacMillan, P J Morrison, N C Nevin, D J Shaw, P S Harper, O W J Quarrell, \\ R G Snell
}

\begin{abstract}
Benign hereditary chorea (BHC) is a rare autosomal dominant disorder characterised by the onset of non-progressive chorea in childhood and the absence of cognitive impairment. Using primers flanking the (CAG)n repeat in IT15, expansion of which is associated with $\mathrm{HD}$, we have detected an abnormal PCR product in four affected members from one family where affected subjects were originally reported to have BHC. The expanded allele contains 38 repeats in the affected parent and this undergoes further enlargement to 39 and 45 repeats in the two affected offspring. We conclude that the diagnostic criteria for BHC should include a normal result from analysis for the (CAG)n expansion identified in HD.
\end{abstract}

(f Med Genet 1993;30:1012-13)

The recent identification ${ }^{1}$ in a gene (IT15) located at $4 \mathrm{p} 16.3$ of the expansion of a trinucleotide repeat (CAG)n sequence in subjects affected by Huntington's disease (HD) prompted us to re-examine families where the movement disorder had been labelled benign hereditary chorea (BHC) rather than HD. Quarrell et $a l^{2}$ reported five families examined for evidence of linkage between the disease and the locus D4S10 (probe G8), concluding that BHC and Huntington's disease (HD) were not allelic. We have reported our findings that in four of these five families there were no expanded repeat sequences in the Huntington's disease gene. ${ }^{3}$ In this paper we report the clinical and molecular findings in the single family where expansions in the repeat sequence were identified.

J C MacMillan
P S Harper

P S Harper

R G Snell

Department of

Medical Genetics,

Belfast City Hospital,

Lisburn Road, Belfast

BT9 7AB, UK.

P J Morrison

NC Nevin

Centre for Human

Genetics, 117

Manchester Road

Sheffield S10 5DN,

UK.

O W J Quarrell

Correspondence to Dr MacMillan.

Received 23 July 1993

Revised version accepted 27 September 1993.

Methods

Affected subjects in the family had been examined clinically by the authors PJM and $\mathrm{NCN}$ and the molecular findings using probe G8 have been reported previously. ${ }^{2}$

Molecular analysis was carried out using primers flanking the polymorphic (CAG)n repeat, as reported previously. ${ }^{14}$ For accurate length measurements, aliquots of the PCR reactions were separated on $4 \%$ denaturing polyacrylamide gels along with sequence markers. Alleles were sized under the assumption that products could vary only by multiples of 3 base pairs, that is, perfect repeats.

\section{Clinical details}

The pedigree is shown in fig 1 . All persons are identified as females, except where molecular data are available on both subjects where the disease is transmitted from father to child. This allows relative anonymity for the family but permits us to display data on any sex differences in the meiotic instability of the mutation.

Subject I 1 was reported to have developed facial twitching in childhood. No other clinical details are known and death was at 75 years.

II 1 developed facial twitching in adolescence and was said to have always been 'fidgety'. Examination showed some dysarthria, a wide based gait, and some finger-nose and heel-shin incoordination. There is no evidence of cognitive decline nor progression of symptoms at 85 years of age.

II 2 had a long history of 'benign' chorea until at the age of 66 progressive chorea and muscle wasting developed. At the age of 71 this subject showed minimal evidence of cognitive decline.

III. 1 is reported to have always been 'fidgety'. By the age of 56, there had been no progression of the chorea but there was evidence of minimal limb and gait ataxia and dysarthria.

III 2 had always tended to be clumsy, tripping a lot as a child. From the age of 35 increasing unsteadiness of gait and dysarthria had become evident but memory and intellect were preserved. Examination at the age of 39 found evidence of cerebellar degeneration 
$\begin{array}{lllll}\| 1 & \| 1 & \| \cdot 2 & \| I \cdot 1 & \| I \cdot 2\end{array}$

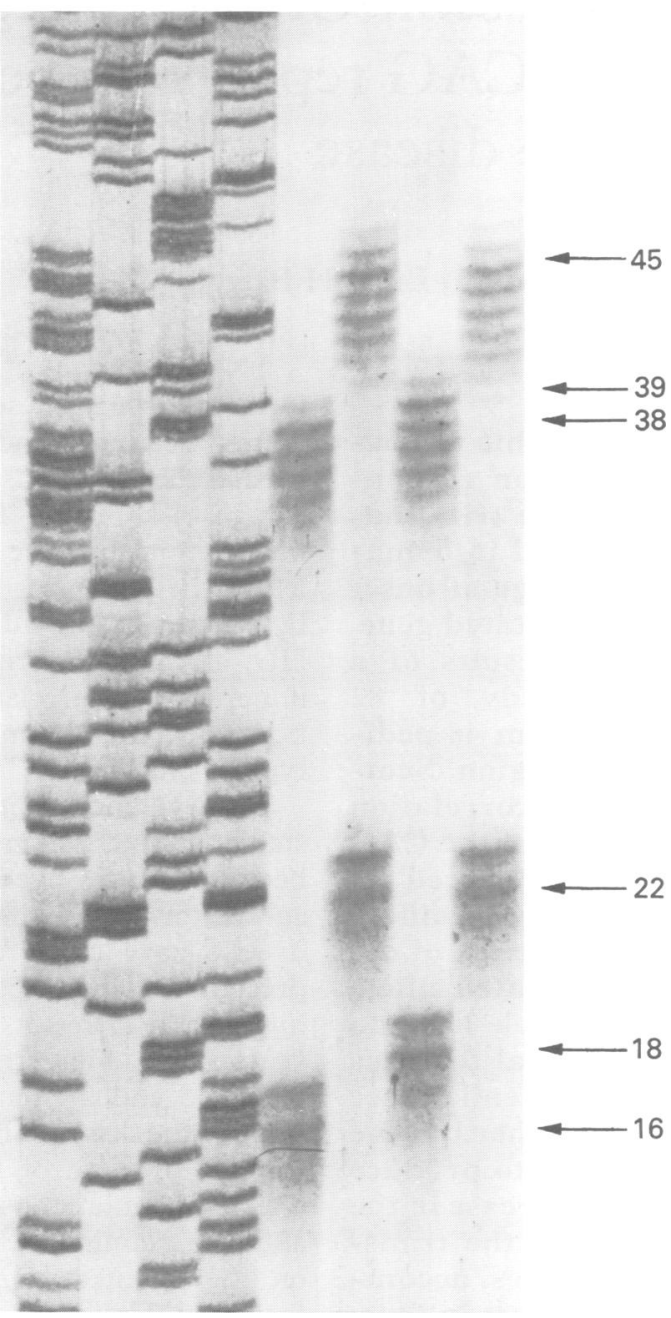

Figure 2 Lanes 1 to 4 show the sequencing ladder used to size the alleles. Lanes 5 to 8 correspond to the subjects shown in the pedigree. The alleles are labelled with the appropriate repeat number calculated from the sequencing ladder.

(limb and gait ataxia) in addition to choreiform movements in the limbs.

\section{Results}

Fig 2 shows the results of the molecular analysis of the CAG repeat sequence in the HD gene in affected subjects in this family. Subject II 1 has CAG repeat lengths of 38 and 16; the disease associated allele undergoes expansion to 39 repeats in III 1 and 45 in III 2 . Subject II. 2 also has 45 copies of the repeat on the disease associated allele.

\section{Discussion}

The clinical diagnosis for affected subjects in this family was initially thought to be benign hereditary chorea in view of the early onset and apparently non-progressive nature of their movement disorder. In addition there was no evidence of cognitive decline in the oldest surviving affected subject at 85 years of age. The progression, from the age of 35 , of the disorder in subject III.2 after the onset of symptoms in childhood prompted clinical doubt as to the exact nature of the disease in this family. The original DNA analysis ${ }^{2}$ could not exclude linkage to $4 \mathrm{p} 16$ in this family because of a combination of small family size and uninformativeness of the G8 typing. The identification in subjects with Huntington's disease of expansion of the trinucleotide repeat sequence in the IT15 gene and the availability of a specific molecular test has enabled us to resolve the diagnostic uncertainty in this family. The results illustrate the unstable nature of the expansion and it is of relevance that the transmitting parent in each case is the father. $\mathrm{We}^{4}$ and others ${ }^{5}$ have shown previously that there is significantly greater variability in the repeat length in the affected offspring of affected fathers with Huntington's disease compared with offspring of affected mothers. The two subjects (II 2 and III 2 ) with progressive disease are also those with the longest repeats (45 in both). There is, however, 31 years' difference in the ages at which the disease in these subjects became progressive suggesting that other factors modify the progression and confirming that repeat length is not of value in predicting age at disease onset. ${ }^{3}$

The application of this molecular genetic test in the assessment of movement disorders in general neurological practice, and more especially in psychiatric practice, will have significant impact on diagnostic accuracy in many such difficult clinical situations.

This work has been supported by the Medical Research Council and the Hereditary Disease Foundation. JCM is supported by the Muscular Dystrophy Group of Great Britain and Northern Ireland. PJM is supported by a Royal College of Physicians of Ireland Glaxo Fellowship in Molecular Biology.

1 The Huntington's Disease Collaborative Research Group. A novel gene containing a trinucleotide repeat that is expanded and unstable on Huntington's disease chromosomes. Cell 1993;72:971-83.

2 Quarrell OWJ, Youngman S, Sarfarazi M, Harper PS. Absence of close linkage between benign hereditary chorea and the locus D4S10 (probe G8). 7 Med Genet and the locus

3 MacMillan JC, Snell RG, Tyler A, et al. Molecular analysis and clinical correlations of the Huntington's disease mutation. Lancet 1993;342:954-8.

4 Snell RG, MacMillan JC, Cheadle JP, et al. Relationship between trinucleotide repeat expansion and phenotypic variation in Huntington's disease. Nature Genet 1993;4:393-7.

5 Duyao M, Ambrose C, Myers R, et al. Trinucleotide repeat length instability and age of onset in Huntington's disease. Nature Genet 1993;4:387-92. 